\title{
Automated Classification of Dementia Using PSO based Least Square Support Vector Machine
}

\author{
T. R. Sivapriya, A. R. Nadira Banu Kamal, and V. Thavavel
}

\begin{abstract}
Machine learning techniques are widely used now for neuro-imaging based diagnosis. These methods yield fully automated clinical decisions, unbiased by variable radiological expertise. This research paper compares and evaluates the performance and reliability of conventional Least Square Support Vector Machine (LSSVM) with that of Particle Swarm Optimization (PSO) based LSSVM in the diagnosis of dementia. The manual interpretation of large volume of brain MRI and cognitive measures may lead to incomplete diagnosis. The PSO-LSSVM approach is trained with multiple biomarkers to facilitate effective, accurate classification which is a requirement of the hour. Wavelet based texture features and multiple biomarkers are fed as input to the classifier. PSO-LSSVM yields $98 \%$ accurate results and outperforms LSSVM classifier in terms of sensitivity, specificity and accuracy in this analysis
\end{abstract}

Index Terms - Classification, dementia, least square support vector machine, particle swarm optimization.

\section{INTRODUCTION}

Medical data and neuroimaging has increasingly employed techniques from machine learning and computer aided diagnostics. For instance, a set of training input data is made to yield a desired output by means of a supervised machine learning algorithm that is "trained" for the purpose. Automated classification methods are commonly used for the analysis of neuroimaging studies. Several multi-resolution approaches have been proposed to detect significant changes in the brain volume using neighbourhood information. Various computer-aided techniques have been proposed in the past and include the study of texture changes in signal intensity [1], grey matter (GM) concentration differences [2], atrophy of sub-cortical limbic structures [3]-[5], and general cortical atrophy [6]-[8].

Brain image analyses have widely relied on univariate voxel-wise analyses, such as voxel-based morphometry (VBM) for structural MRI [9]. In such analyses, brain images are first spatially registered to a common stereotaxic space, and then mass univariate statistical tests are performed in each voxel to detect significant group differences. However, the sensitivity of these approaches is limited when the differences are spatially complex and involve a combination of different voxels or brain structures [10]. Recently, there has been a growing interest in support vector machines (SVM)

Manuscript received December 30, 2012; revised April 11, 2013.

T. R. Sivapriya is with the Department of Computer Science, Lady Doak College, Madurai, Tamilnadu, India. (e-mail:spriya.tr@gmail.com).

A. R. Nadira Banu Kamal is with the Department of MCA, T.B.A.K. College for Women, Kilakarai, Ramanathapuram,Tamilnadu, India (e-mail: nadira_kamal@hotmail.com).

V.Thavavel is with the Department of MCA, Karunya University, Coimbatore, Tamilnadu, India (e-mail: vthavavel@karunya.edu). methods[11][12] to overcome the limits of these univariate analyses. There are some studies that involved neuropsychological measures for the diagnosis of dementia and the progression from MCI (Mild Cognitive Impairment) to Alzhemier Dementia. Reference [13] states that MMSE (Mini Mental State Examination) and CVLT-LDTR (California Verbal Learning Test Long Delayed Total Recall) were the only measures that arose from such multivariate analysis as independently associated with progression risk from MCI to early AD. According to their results, subjects scoring below 26/30 on MMSE and 4/16 on CVLT-LDTR constitute a MCI subgroup at high risk of progressing to early AD.

MMSE is one of the most used tests for screening of cognitive impairment worldwide, and it has been reported that decline in MMSE starts approximately three years before the diagnosis of dementia [14]. Other tests used to determine general cognitive status (ADAS-cog, Adden brooke's Cognitive Examination) have been reported as good predictors, independently or associated with other neuropsychological and neuroimaging measures [15][16]. Alzhemier's dementia(AD) is more prevalent today. The brain volume is significantly changed in Alzhemier's Dementia patients compared to healthy subjects of the same age group. Visual assessment of ventricle volume or shape change has shown to be quite reliable [17][18] in detecting AD. Fourier analysis applied for image features[19], [20], application of wavelets[21], Haralick components [22] are applied to extract features for brain MRI analysis.

Research papers with different approaches for image classification and segmentation are reported in the literature. This study sets out to investigate the reliability and efficiency of PSO based LSSVM techniques in detecting demented and non-demented patients combining MMSE and Clinical Dementia Ratio (CDR) with brain volume. To achieve a high degree of success in treatment, one requirement is to establish proper classification and diagnosis of patients with complex neurological diseases. Hence a central task is to discover most common features specific to each pathologic state (e.g. normal vs. demented) or differential profiles between experimental conditions from high dimensional data [23].

\section{A. Related Work}

There are several studies that demonstrate cross-sectional inter-group differences associated with Alzhemier's Dementia (AD). Fully automatic brain tissue classification from magnetic resonance images (MRI) is of great importance for research and clinical studies of the normal and diseased human brain. Several types of medical image segmentation methods can be applied to anatomical brain MRI. Previous work on MR image based detection of dementia, or more specifically Alzheimer's disease (AD), has 
mainly focussed on the hippocampal shape. The work in [24] is most related to ours. They segment the MR brain images into grey matter, white matter, and cerebrospinal fluid. After registration to a population template, the (whole-brain) grey matter segmentations are used as feature vectors in a support vector machine (SVM). Leave-one-out validation on a group of 40 subjects suffering from mild to moderate dementia and 40 normal controls resulted in an accuracy of $92 \%$. Automatically segmented hippocampi, which are registered to each other [25] is used in order to construct a dissimilarity matrix based on the deformation metric implied by their diffeomorphic registration approach. As in [26] a new method was proposed for abnormal MRI volume identification with slice segmentation using Fuzzy C-means (FCM) algorithm. Automated Segmentation and Classification of brain MRI [27] in which an SVM classifier was used for normal and abnormal slices classification with statistical features was proposed. The remainder of the paper is organized as follows: Section II focuses on SVM and LSSVM classifiers, Section III describes the proposed Methodology and the Data Set used for the study Section IV discusses the Results.

\section{OVERVIEW OF CLASSIFIER}

\section{A. Support Vector Machines}

Support Vector Machines are new learning techniques that were introduced in 1995 by Vapnik [28]. In terms of theory the SVMs are well founded and proved to be very efficient in classification tasks. Support Vector Machines (SVMs) are feed-forward networks with a single layer of nonlinear units.

Their design has good generalization performance as an objective and follows for that reason the principle of structural risk minimization that is rooted in VC dimension theory. Those training points for which the equality of the separating plan is satisfied (i.e.) $\mathrm{y}_{\mathrm{i}}\left(\mathrm{x}_{\mathrm{i}} \cdot \mathrm{w}+\mathrm{b}\right) \geq 0$ for all $\mathrm{i}$, those which wind up lying on one of the hyperplanes $H_{l}, H_{2}$, and whose removal would change the solution found, are called Support Vectors (SVs). This algorithm is firmly grounded in the framework of statistical learning theory - Vapnik Chervonenkis (VC) theory, which improves the generalization ability of learning machines to unseen data [29], [30]. In the last few years Support Vector Machines have shown excellent performance in many real-world applications including object recognition [31], and face detection, dementia diagnosis [32] in images.

\section{B. LSSVM}

The least squares support vector machine (LSSVM) is a least squares version of support vector machine (SVM). In this version one finds the solution by solving a set of linear equations instead of a convex quadratic programming (QP) for classical SVMs. Least Squares SVMs (LSSVMs) classifiers, was proposed by Suykens and Vandewalle. LSSVM is a class of kernel based learning method. Primary goals of the LSSVM models are regression and classification. LSSVM is a regularized supervised approximator, which has been proved to be efficient for function approximation. Only solving linear equation is needed in the optimization process, which not only simplifies the process, but also avoids the problem of local minima in SVM. In this section, a short summary of the LSSVM model is given. The LSSVM model [33] is defined in its primal weight space by,

$$
\hat{y}(x)=\omega^{T} \varphi(x)+b
$$

where $\varphi(x)$ is a function which maps the input space into a higher dimensional feature space, $x$ is the $M$-dimensional vector of inputs $x_{j}$, and $\omega$ and $b$ the parameters of the model. Least Squares Support Vector Machines for function estimation formulate the following optimization:

$$
\min _{w, b, e} J(\omega, e)=\frac{1}{2} \omega^{T} \omega+\gamma \frac{1}{2} \sum_{i=1}^{N} e_{i}^{2}
$$

subject to

$$
y^{i}=\omega^{T} \varphi\left(x^{i}\right)+b+e^{i}, i=1, \ldots N
$$

The parameter set $\theta$ consists of vector $\omega$ and scalar $b$. Solving this optimization problem in dual space leads to finding the $\alpha_{i}$ and $b$ coefficients in the following solution:

$$
h(x)=\sum_{i=1}^{N} \alpha{ }_{i} K\left(x, x_{i}\right)+b
$$

Function $K\left(x, x_{i}\right)$ is the kernel defined as the dot product between the $\phi(x) T$ and $\phi(x)$ mappings. The meta-parameters of the LSSVM model are the width of the Gaussian kernels and $\gamma$ the regularization factor.

\section{Particle Swarm Optimisation}

Particle Swarm Optimisers (PSO) is a new trend in evolutionary algorithms, being inspired in group dynamics and its synergy. In PSO individuals are called particles and the population is called a swarm. PSO are inspired in the intelligent behaviour of beings as part of an experience sharing community as opposed to an isolated individual reactive response to the environment. PSO algorithm is as follows:

1) Initialize a population size, positions and velocities of agents, and the number of weights and biases.

2) The current best fitness achieved by particle $p$ is set as pbest. The pbest with best value is set as gbest and this value is stored.

3) Evaluate the desired optimization fitness function $f p$ for each particle as the Mean Square Error (MSE) over a given data set.

4) Compare the evaluated fitness value $f p$ of each particle with its pbest value. If $f p<$ pbest then pbest $=f p$ and best $x p=x p, x p$ is the current coordinates of particle $p$, and bestxp is the coordinates corresponding to particle $p$ 's best fitness so far.

5) The objective function value is calculated for new positions of each particle. If a better position is achieved by an agent, pbest value is replaced by the current value. If $f p<$ gbest then gbest $=p$, where gbest is the particle having the overall best fitness over all particles in the swarm.

6) Change the velocity and location of the particle based on random number assigned.

$$
\begin{gathered}
\text { vid }=v i d+\varphi 1 \times r n d() \times(\text { pid-xid })+\varphi 2 \times r n d() \times(\text { pgd-xid }) \\
x i d=x i d+v i d ;
\end{gathered}
$$

where $i$ is the particle, $\varphi 1, \varphi 2$ are learning rates governing 
the cognition and social components, $g$ represents the index of the particle with the best p-fitness, and $d$ is the dth dimension.

\section{Methodology}

\section{A. Data Preprocessing}

Data is pre-processed by applying z-score normalization. Normalization is a process where the attribute data are scaled so as to fall within a small specified range 0.0 to 1.0 . In $\mathrm{Z}$-score normalization, the values for an attribute ' $\mathrm{A}$ ' are normalized based on the mean and standard deviation of $\mathrm{A}$. This method of normalization is useful when the actual minimum and maximum of attribute $A$ are unknown, or when there are outliers that dominate the min-max normalization.

\section{B. Feature Extraction}

In this paper, the purpose is to individually classify $\mathrm{AD}$ patients and healthy elderly control subjects by using a whole-brain MR image analysis. A texture based analysis of the MR images employing wavelets along with Haralick methods is used to extract feature parameters. Thereby, focus is on characteristics of the distribution of the gray matter (GM), white matter (WM), and cerebrospinal fluid (CSF), which intuitively makes sense when dealing with neurodegenerative diseases in general and AD in particular. Steps for feature extraction are as follows:

1) The voxel-wise texture features of image I are extracted at each slice of 3D ROI by convoluting with 2D Gabor filters [34] and averaging inside the ROI. Haralick features like inertia, entropy and correlation are calculated for each training image and the average is computed at different angles.

2) Compute the overall mean of each feature of the entire set of images considered for feature extraction.

3) Feature reduced vectors generated by wavelets is given as input vectors to the classifier.

\section{C. $L S S V M-P S O$}

For the standard SVMs and its reformulations, LSSVM, the regularization parameter and kernel parameters are called hyper-parameters, which play a crucial role to the performance of the SVMs. There exist different techniques for tuning the hyper-parameters related to the regularization constant and the parameter of kernel function. Analytical and algebraic methods can be used for tuning the parameters. Error measure is the central theme of analytical and algebraic techniques. However nowadays genetic algorithm and evolutionary strategy are employed for the hyper-parameters of SVMs. Grid search method involves a costly search procedure and gradient-based methods do not provide optimum performance measure. PSO is an evolutionary computation techniques based on swarm intelligence. It has many advantages over other heuristic techniques. This technique can exploit the distributed and parallel computing capabilities, to escape local optima and quick convergence.

\section{Optimization of LSSVM Parameters}

In the case of the algorithm LSSVM with radial kernel function [35], optimized parameters are: $\gamma$, which is the weight at which the testing errors will be treated in relation to the separation margin and parameter $\sigma$, which corresponds to the width of the kernel function. It is not known in advance what combination of these two parameters will achieve the best result of classification. It is impossible to complete the search space of models, therefore the choice of optimal set of parameters is a very complex problem, and the way its solution is a key element of the classification system. In order to find the best values several techniques like Grid-Search [36], K-fold Cross-Validation, Particle Swarm Optimization [37[-[42] are used. PSO provides better optimization than Grid-Search and K-fold method.

\section{E. Data Set}

OASIS provides brain imaging data that are freely available for distribution and data analysis. This data set consists of a cross-sectional collection of 416 subjects covering the adult life span aged 18 to 96 including individuals with early-stage Alzheimer's Disease (AD) . For each subject, 3 or 4 individual T1-weighted MRI scans obtained within a single imaging session are included. Table I gives the list of MRI features considered for the study.

\begin{tabular}{|c|c|}
\hline Variable & Definition \\
\hline $\mathrm{X}_{1}$ & Age \\
\hline $\mathrm{X}_{2}$ & Education \\
\hline $\mathrm{X}_{3}$ & Clinical Dementia Rating \\
\hline $\mathrm{X}_{4}$ & Total intracranial volume \\
\hline $\mathrm{X}_{5}$ & Whole brain volume \\
\hline $\mathrm{X}_{6}$ & Mini Mental State Examination \\
\hline
\end{tabular}

\section{1) Division of Data into Training and Testing}

When a classification algorithm is developed, it is important to know that the classifier works well enough to be useful for the application. It is easy to design an optimistically biased (low error, over-trained) algorithm. If, for example, the same data is used for model selection (i.e. optimization of $C$ and $\gamma$ or $n$ in our case), model training, and validation, an obvious risk is the creation of a machine which does not generalize. Such a model may be of limited effectiveness for classifying novel data. To eliminate such circular logic for this study, validation is performed on independent or unseen data.

The records of 370 patients with Alzhemier's disease (AD) Cognitively Normal $(\mathrm{CN})$ datasets were randomly divided into two sets of $75 \mathrm{AD}, 75 \mathrm{CN}$ for training and 50AD, $50 \mathrm{CN}$ for testing. The training datasets need not be exactly age and gender matched if age and gender are given as input to the SVM.

TABLE II: DATA SET FOR CLASSIFICATION

\begin{tabular}{|c|c|c|c|c|}
\hline Dataset & \multicolumn{2}{|c|}{ Longitudinal } & \multicolumn{2}{c|}{ Crosssectional } \\
\hline & Demented & $\begin{array}{c}\text { Cognitively } \\
\text { Normal }\end{array}$ & Demented & $\begin{array}{c}\text { Cognitively } \\
\text { Normal }\end{array}$ \\
\hline Training & 75 & 75 & 75 & 75 \\
\hline Testing & 50 & 50 & 50 & 50 \\
\hline
\end{tabular}

\section{2) Cross validation of the Classifiers}

Cross Validation is a statistical analysis method used to verify the performance of classifiers. The basic idea is that the original dataset is divided into training datasets which are used for training classifiers, and validation datasets for 
testing the trained models to obtain the classification accuracy as the evaluation performance of classifiers. This paper uses Leave-One-Out Cross Validation.

\section{RESULT}

All classification results could have an error rate and on occasion will either fail to identify dementia or misclassify a normal patient as demented. It is common to describe this error rate by the terms true positive and false positive and true negative and false negative as follows:

True Positive (TP): the classification result is positive in the presence of the clinical abnormality.

True Negative (TN): the classification result is negative in the absence of the clinical abnormality.

False Positive (FP): the classification result is positive in the absence of the clinical abnormality.

False Negative (FN): the classification result is negative in the presence of the clinical abnormality.

Sensitivity $=\mathrm{TP} /(\mathrm{TP}+\mathrm{FN}) \times 100 \%$

Specificity $=\mathrm{TN} /(\mathrm{TN}+\mathrm{FP}) \times 100 \%$

Accuracy $=(\mathrm{TP}+\mathrm{TN}) /(\mathrm{TP}+\mathrm{TN}+\mathrm{FP}+\mathrm{FN}) \times 100 \%$

TP, TN, FP, FN, Sensitivity, Specificity and Accuracy are used to measure the performance of the classifiers. The PSO-LSSVM method was tested on longitudinal data divided into two groups. Classification rates of $95 \%$ and $97 \%$ were achieved. An improved accuracy of $96 \%$ was obtained by taking into account only a volume of interest i.e. the grey matter. However the results are further enhanced only if multiple biomarkers including age, clinical dementia rating and MMSE are included.

A leave-one-out test on a set of 50 healthy controls and 50 patients with AD resulted in a classification accuracy of $96 \%$. The experiment involved a training stage on the full set though. Table III and Table IV depict the result for the classification of longitudinal and cross-sectional data with SVM, LSSVM and hybrid PSO-LSSVM.

TABLE III: COMPARISON OF EFFICIENCY OF CLASSIFIERS FOR LONGITUDINAL DATA SET

\begin{tabular}{|l|l|c|c|}
\hline MEASURE & SVM & LSSVM & PSO-LSSVM \\
\hline Sensitivity \% & 89 & 92 & 95 \\
\hline Accuracy \% & 90 & 90 & 96 \\
\hline Specificity \% & 89 & 93 & 97 \\
\hline
\end{tabular}

TABLE IV: COMPARISON OF EFFICIENCY OF CLASSIFIERS FOR CROSS-SECTIONAL DATA SET

\begin{tabular}{|l|c|c|c|}
\hline MEASURE & \multicolumn{3}{|c|}{ CLASSIFIER } \\
\hline & SVM & LSSVM & PSO-LSSVM \\
\hline Sensitivity \% & 92 & 94 & 98 \\
\hline Accuracy \% & 89 & 90 & 96 \\
\hline Specificity \% & 90 & 92 & 95 \\
\hline
\end{tabular}

The results of Table $\mathrm{V}$ indicate that the classification accuracy increases as the cognitive measures are added. Although initially MMSE was used as a screening test for dementia, it can be effectively used as a feature for classification of dementia using machine learning methods.
TABLE V: COMPARISON OF EFFICIENCY OF CLASSIFIERS FOR LONGITUDINAL DATA SET WITH PSO-LSSVM WITH VARYING FEATURES

\begin{tabular}{|l|c|c|c|}
\hline \multirow{2}{*}{$\begin{array}{c}\text { MEASURE OF } \\
\text { PERFORMANCE }\end{array}$} & \multicolumn{3}{|c|}{ CLASSIFIER - PSO-LSSVM } \\
\cline { 2 - 4 } & MRI-feature & MMSE & CDR \\
\hline Sensitivity \% & 89 & 94 & 98 \\
\hline Accuracy \% & 93 & 92 & 96 \\
\hline Specificity \% & 90 & 91 & 95 \\
\hline
\end{tabular}

\section{CONCLUSION}

In summary, the results are in line with those reported in the literature, taking into account that multiple biomarkers are included as inputs for the classifier. It can be said that PSO-LSSVM is a promising classifier to obtain a superior prediction performance for automated diagnosis of dementia. It is observed that significant improvements in the performance of the algorithm can be realised through optimisation by PSO.

Classification accuracy is improved when the neuro-imaging data is combined with MMSE and CDR values. The results do suggest though that it could be beneficial to additionally take into account all neuropsychological measures as input. This aspect can be investigated in future work, by testing the method for different combination of biomarkers that can improve the accuracy of the PSO-LSSVM classifier.

\section{ACKNOWLEDGMENT}

I would like to acknowledge the guidance, and expert opinion given by Dr. Sabesan, former Prof. and Head, Govt. Rajaji Hospital, Madurai.

\section{REFERENCES}

[1] P. A. Freeborough and N. C. Fox , "MR image texture analysis applied to the diagnosis and tracking of Alzheimer's disease," IEEE Trans. Med. Imag., vol. 17, no. 3, pp. 475-479, 1998.

[2] G. B. Frisoni, C. Testa, A. Zorzan, F. Sabattoli, A. Beltramello, H. Soininen, and M. P. Laakso, "Detection of grey matter loss in mild alzheimer's disease with voxel based morphometry," J. Neurology, Neurosurgery \& Psychiatry, vol. 73, pp. 657-664, 2002.

[3] P. M. Thompson, K. M. Hayashi et al., "Mapping hippocampal and ventricular change in alzheimer disease," Neurolmage, vol. 22, pp. 1754-1766, 2004.

[4] G. B. Frisoni, F. Sabattoli, A. D. Lee, R. A. Dutton, A. W. Toga, and P. M. Thompson, "In vivo neuropathology of the hippocampal formation in AD: A radial mapping MR-based study," NeuroImage, vol.32, pp. 104-10, 2006.

[5] J. G. Csernansky, L.Wang et al., "Early DAT is distinguished from aging by high-dimensional mapping of the hippocampus. Dementia of the alzheimer type," Neurology, vol. 55, pp. 1636-1643, 2000.

[6] P. M. Thompson, K. M. Hayashi et al., "Dynamics of gray matter loss in Alzheimer's disease," Journal of Neuroscience, vol. 23, pp. 994-1005, 2003.

[7] D. Chan, J. C. Janssen, J. L. Whitwell et al., "Change in rates of cerebral atrophy over time in early-onset alzheimer's disease: Longitudinal MRI study," Lancet, vol. 362, pp. 1121-1122, 2003.

[8] J. P. Lerch, J. C. Pruessner, A. Zijdenbos, H. Hampel, S. J. Teipel, and A. C. Evans, "Focal decline of cortical thickness in alzheimer's disease identified by computational neuroanatomy," Cerebral Cortex, vol. 15, pp. 995-1001, 2005.

[9] J. Ashburner and K. J. Friston, "Voxel-based morphometry-the methods," NeuroImage, vol. 11, no. 6, pp. 805-21, 2000.

[10] C. Davatzikos, "Why voxel-based morphometric analysis should be used with great caution when characterizing group differences," NeuroImage, vol. 23, no. 1, pp. 7-20, 2004. 
[11] B. Magnin, L. Mesrob et al., "Support vector machine-based classification of Alzheimer's disease from whole-brain anatomical MRI," Neuroradiology, vol. 5, no. 1, pp. 73-83, 2009.

[12] B. Scholkopf and A .J. Smola, Learning with Kernels, MIT Press, 2001.

[13] A. Pozueta, E. Rodriguez-Rodriguez et al., "Detection of early Alzheimer's disease in MCI patients by the combination of MMSE and an episodic memory test," BMC Neurology, vol. 11, pp.78, 2011.

[14] Amieva H, Le Goff M et al., "Prodromal Alzheimer's disease: successive emergence of the clinical symptoms," Annals of Neurology, vol. 64, pp. 492-498, 2008.

[15] J. Mitchell, R. Arnold, K. Dawson, P. J. Nestor, J. R. Hodges, "Outcome in subgroups of mild cognitive impairment (MCI) is highly predictable using a simple algorithm," Journal of Neurology, vol. 256, pp.1500-1509, 2009.

[16] M. Ewers et al., "Prediction of conversion from mild cognitive impairment to Alzheimer's disease dementia based upon biomarkers and neuropsychological test performance," Neurobiology of Aging, vol. 33, no. 7, pp. 1203-1214, 2012.

[17] Mahmoud-Ghoneim, D. Toussaint et al., "Three dimensional texture analysis in MRI: a preliminary evaluation in gliomas," Magnetic Resonance Imaging, vol. 21, pp. 983-87, 2003.

[18] Snyder, AZ., Girton, LE., Morris, JC., Buckner, RL. , "Normative estimates of cross-sectional and longitudinal brain volume decline in aging and AD," Neurology, vol. 64, pp. 1032-1039, 2009.

[19] H. Zhu, , B. G. Goodyear et al., "A new local multiscale Fourier analysis for medical imaging," Medical Physics, vol. 30, pp 1134-1141, 2003.

[20] Ferrarini, W. M. Palm, H. Olofsen, M. A. Van Buchem, J. H. Reiber, F. Admriraal-Behloul, "Shape Differences of the brain ventricles in Alzheimer's Disease," Neuroimage, vol. 32, pp. 1060-9, 2006.

[21] S. Chaplot, L. M. Patnaik, and N. R. Jagannathan, "Classification of magnetic resonance brain images using wavelets as input to support vector machine and neural network," Biomedical Signal Processing and Control, pp. 86-92, 2006.

[22] H. Selvaraj, S. ThamaraiSelvi, D. Selvathi, and L. Gewali1, "Brain MRI Slices Classification Using Least Squares Support Vector Machine," IC-MED, vol. 1, no. 1, pp. 21- 33, 2007.

[23] M. Bozzali et al., "The contribution of voxel-based morphometry in staging patients with mild cognitive impairment," Neurology, vol. 67, pp.453-460, 2006.

[24] S. Kloppel, CM. Stonnington, C. et al., "Automatic classification of MR scans in Alzheimer's disease," Brain, vol. 131, pp. 681-689, 2008.

[25] M. I. Miller et al., "Collaborative computational anatomy - an MRI morphometry study of the human brain via diffeomorphic metric mapping," Human Brain Mapping, vol. 30, no. 7, pp. 2132-2141, 2009.

[26] M. C. Clark, L. O. Hall, D. B. Goldgof, L. P. Clarke, R. P. Velthuizen, M.S. Silbiger, "MRI Segmentation using Fuzzy Clustering Techniques," IEEE Engineering in Medicine and Biology, pp. 730-742, 1994.

[27] L. Antonie, "Automated Segmentation and Classification of Brain Magnetic Resonance Imaging," C615 Project .

[28] V. N. Vapnik, The Nature of Statistical Learning Theory, Springer-Verlag, New York, 1995.

[29] C. J. C. Burges, Tutorial on support vector machines for pattern recognition. Data Mining and Knowledge Discovery, 1998, vol. 2, no. 2, pp. $955-974$.

[30] C. J. C. Burges and B. Scholkopf, "Improving the accuracy and speed of support vector learning machines. In M. Mozer, M. Jordan, and T. Petsche, editors, Advances in Neural Information Processing Systems, ch. 9, MIT Press.

[31] M. Pontil and A. Verri, "Support vector machines for 3-D object recognition," IEEE Trans. Pattern Anal. Machine Intelligence, 1998, vol. 20, pp. 637-646.

[32] T. R. Sivapriya, V. Saravanan, Dementia," Diagnosis relying on Texture based features and SVM classification," ICGST Artificial Intelligence and Machine Learning, vol. 11, no. 1, pp. 9-19, 2011.

[33] J. A. K. Suykens, T. Van Gestel, J. De Brabanter, B. De Moor and J. Vandewalle, Least Squares Support Vector Machines, World Scientific Publishing Company, 2002.
[34] Bagci, U., Li Bai, A., "Detecting Alzheimer Disease in Magnetic Resonance Brain Images Using Gabor Wavelets," in Proc.IEEE 15th International Conference on Signal Processing and Communications Applications, Eskisehir, Turkey, pp. 1- 4, 2007.

[35] C. Cortes and V. Vapnik, "Support-vector network," Machine Learning, vol. 20, pp. 273-297, 1995.

[36] C.-W. Hsu, C.-C. Chung, C.-J. Lin. (March 13, 2010). A Practical Guide to Support Vector Classification," National Taivan University. [Online]. Available: www.csie.ntu.edu.tw/cjlin.

[37] S. Das, A. Abraham, and A. Konar, Particle Swarm Optimization and Differential Evolution Algorithms: Technical Analysis, Applications and Hybridization Perspectives, Berlin: Springer, 2008.

[38] J. Keneddy and R. C. Eberhart, "Particle swarm optimization," in Proc. IEEE Int. Conf. Neural Netw., Piscataway, New York, pp. 1942-1948, 1995.

[39] M.-Z. Lu, C. L. Philip Chen, J.-B. Huo, "Optimization of combined kernel function for SVM by particle swarm optimization," in Proc. Eighth Int. Conf. Machine Learning Cybernet., Baoding, China, pp. 1160-1166, 2009

[40] M. G. H. Omran, "Particle swarm optimization methods for pattern recognition and image processing," Ph.D. thesis, University of Pretoria, 2004.

[41] C. Sun and D. Gong, "Support Vector Machines with PSO Algorithm for Short-Term Load Forecasting," in Proc. IEEE Int. Conf. Netw., Sensing Contr. ICNSC '06, Ft. Lauderdale, USA, pp. 676-680,2006.

[42] Q.-Z. Yao, J.eCai, J.-L. Zhang, "Simultaneous feature selection and LSSVM parameters optimization algorithm based on PSO," in Proc. World Congr. Comput. Sci. Informa. Engin. CSIE 2009, LosAngeles, USA, pp. 723-727, 2009.

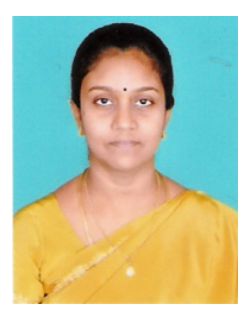

T. R. Sivapriya received her B.Sc. Computer Applications, Master of Computer Applications and M.Phil. from Madurai Kamaraj University. Currently she is working as asst. professor at Lady Doak College, Madurai. She is pursuing her Ph.D. at Karunya University, Coimbatore under the supervision Dr. A. R. Nadira Banu Kamal and Dr.V. Thavavel. She has presented many research papers in National, International conferences and published papers in International Journals. She has totally 11 years teaching experience and 4 years of Research Experience. Her research interests include Image Processing and Data Mining.

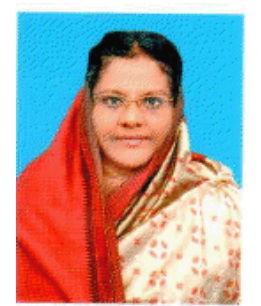

A. R. Nadira Banu Kamal obtained her Bachelor's degree in Mathematics from University of Madras, and Masters Degree in Applied Mathematics from Anna University. She has completed her M.Phil from Alagappa University and obtained her $\mathrm{PhD}$ in Applied Mathematics from Manonmaniam Sundaranar University. She has done many Major and Minor research projects funded by UGC. She has presented many research papers in National, International conferences and Journals. She has totally 25 years of experience in teaching including 8 years as a researcher. She has organized several Seminars, National and International Conferences. Currently, she is serving as the director of MCA department in TBAK College, Kilakarai. At present she is supervising 5 research scholars for their $\mathrm{PhD}$ degree and has guided many M.Phil. scholars.

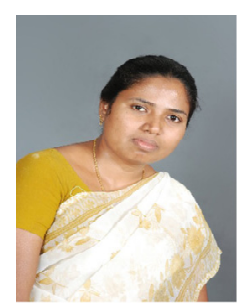

V. Thavavel received her ME degree from Anna University, Trichy and $\mathrm{PhD}$ degree from Madurai Kamaraj University. She is an associate professor and Head of the Department of Computer Applications, Karunya Univeristy, India. She also had the previlege of bagging the overall proficiency award for her BSc and MCA studies. Her research examines the applications of soft-computing and wavelet theories for low dose medical image processing. She has authored or co-authored more than 25 research papers in international journals and conferences. 